\title{
Front Matter: Volume 8451
}

, "Front Matter: Volume 8451," Proc. SPIE 8451, Software and Cyberinfrastructure for Astronomy II, 845101 (24 September 2012); doi: $10.1117 / 12.2006282$

SDIE Event: SPIE Astronomical Telescopes + Instrumentation, 2012, Amsterdam, Netherlands 


\title{
PROCEEDINGS OF SPIE
}

\section{Software and Cyberinfrastructure for Astronomy II}

\author{
Nicole M. Radziwill \\ Gianluca Chiozzi \\ Editors
}

\section{1-4 July 2012}

Amsterdam, Netherlands

Sponsored by

SPIE

Cooperating Organisations

American Astronomical Society (United States) - Netherlands Institute for Radio Astronomy (ASTRON) (Netherlands) • Ball Aerospace \& Technologies Corporation (United States)

Canadian Astronomical Society (CASCA) (Canada) • European Astronomical Society (Switzerland) • ESO_European Southern Observatory (Germany) • International Astronomical Union - Korea Astronomy and Space Science Institute (KASI) (Republic of Korea) • National Radio Astronomy Observatory • POPSud (France) • TNO (Netherlands)

Published by

SPIE 
The papers included in this volume were part of the technical conference cited on the cover and title page. Papers were selected and subject to review by the editors and conference program committee. Some conference presentations may not be available for publication. The papers published in these proceedings reflect the work and thoughts of the authors and are published herein as submitted. The publisher is not responsible for the validity of the information or for any outcomes resulting from reliance thereon.

Please use the following format to cite material from this book:

Author(s), "Title of Paper," in Software and Cyberinfrastructure for Astronomy II, edited by

Nicole M. Radziwill, Gianluca Chiozzi, Proceedings of SPIE Vol. 8451 (SPIE, Bellingham, WA, 2012) Article CID Number.

ISSN: 0277-786X

ISBN: 9780819491527

Published by

SPIE

P.O. Box 10, Bellingham, Washington 98227-0010 USA

Telephone +1 3606763290 (Pacific Time) · Fax +1 3606471445

SPIE.org

Copyright (C 2012, Society of Photo-Optical Instrumentation Engineers.

Copying of material in this book for internal or personal use, or for the internal or personal use of specific clients, beyond the fair use provisions granted by the U.S. Copyright Law is authorized by SPIE subject to payment of copying fees. The Transactional Reporting Service base fee for this volume is $\$ 18.00$ per article (or portion thereof), which should be paid directly to the Copyright Clearance Center (CCC), 222 Rosewood Drive, Danvers, MA 01923. Payment may also be made electronically through CCC Online at copyright.com. Other copying for republication, resale, advertising or promotion, or any form of systematic or multiple reproduction of any material in this book is prohibited except with permission in writing from the publisher. The CCC fee code is 0277-786X/12/\$18.00.

Printed in the United States of America.

Publication of record for individual papers is online in the SPIE Digital Library.

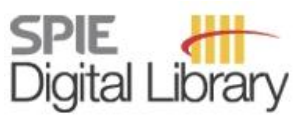

SPIEDigitalLibrary.org

Paper Numbering: Proceedings of SPIE follow an e-First publication model, with papers published first online and then in print and on CD-ROM. Papers are published as they are submitted and meet publication criteria. A unique, consistent, permanent citation identifier (CID) number is assigned to each article at the time of the first publication. Utilization of CIDs allows articles to be fully citable as soon as they are published online, and connects the same identifier to all online, print, and electronic versions of the publication. SPIE uses a six-digit CID article numbering system in which:

- The first four digits correspond to the SPIE volume number.

- The last two digits indicate publication order within the volume using a Base 36 numbering

system employing both numerals and letters. These two-number sets start with $00,01,02,03,04$, $05,06,07,08,09,0 A, 0 B \ldots 0 Z$, followed by 10-1Z, 20-2Z, etc.

The CID Number appears on each page of the manuscript. The complete citation is used on the first page, and an abbreviated version on subsequent pages. Numbers in the index correspond to the last two digits of the six-digit CID Number. 


\title{
Contents
}

\author{
xix Conference Committee \\ xxi The cosmic microwave background: observing directly the early universe (Plenary Paper) \\ [8442-506] \\ P. de Bernardis, S. Masi, Univ. degli Studi di Roma La Sapienza (Italy)
}

\section{Part 1}

\section{INNOVATIONS}

845102 A next-generation open-source toolkit for FITS file image viewing [8451-1]

E. Jeschke, T. Inagaki, R. Kackley, Subaru Telescope, National Astronomical Observatory of Japan (United States)

845103 Data mining and knowledge discovery resources for astronomy in the web 2.0 age [8451-56]

S. Cavuoti, Univ. degli Studi di Napoli Federico II (Italy) and INAF - Osservatorio Astronomico di Capodimonte (Italy); M. Brescia, INAF - Osservatorio Astronomico di Capodimonte (Italy) and Univ. degli Studi di Napoli Federico II (Italy); G. Longo, Univ. degli Studi di Napoli Federico II (Italy) and INAF - Osservatorio Astronomico di Capodimonte (Italy) and California Institute of Technology (United States)

845104 Science ground segment for the ESA Euclid Mission [8451-3]

F. Pasian, INAF - Osservatorio Astronomico di Trieste (Italy); J. Hoar, European Space Astronomy Ctr. (Spain); M. Sauvage, CEA, Lab. AIM (France); C. Dabin, M. Poncet, Ctr. National d'Etudes Spatiales (France); O. Mansutti, INAF - Osservatorio Astronomico di Trieste (Italy)

845105 The VO-Dance web application at the IA2 data center [8451-4]

M. Molinaro, C. Knapic, R. Smareglia, INAF - Osservatorio Astronomico di Trieste (Italy)

SOFTWARE ENGINEERING

845106 Distributed agile software development for the SKA (Invited Paper) [8451-5]

A. Wicenec, The Univ. of Western Australia (Australia); R. Parsons, ThoughtWorks, Inc. (United States); S. Kitaeff, K. Vinsen, C. Wu, The Univ. of Western Australia (Australia); P. Nelson, ThoughtWorks, Inc. (United States); D. Reed, ThoughtWorks, Inc. (Australia)

845107 Evolution of the top level control software of astronomical instruments at ESO [8451-11 1] E. Pozna, ESO, European Organization for Astronomical Research in the Southern Hemisphere (Germany) 
845108 Discovery Channel Telescope software component template and state design: principles and implementation [8451-7]

P. J. Lotz, M. J. Lacasse, R. C. Godwin, Lowell Observatory (United States)

845109 Instrument control software development process for the multi-star AO system ARGOS [8451-8]

M. Kulas, Max-Planck-Institut für Astronomie (Germany); L. Barl, Max-Planck-Institut für extraterrestrische Physik (Germany); J. L. Borelli, W. Gässler, Max-Planck-Institut für Astronomie (Germany); S. Rabien, Max-Planck-Institut für extraterrestrische Physik (Germany)

\section{DATA MANAGEMENT AND PROCESSING}

$84510 A$ Data management for the EVLA (Invited Paper) [8451-9]

B. J. Butler, C. J. Chandler, National Radio Astronomy Observatory (United States)

8451 OB Design and capabilities of the MUSE data reduction software and pipeline [8451-10] P. M. Weilbacher, O. Streicher, T. Urrutia, Leibniz-Institut für Astrophysik (Germany); A. Jarno, A. Pécontal-Rousset, R. Bacon, Observatoire de Lyon, CNRS, Univ. de Lyon (France) and Ecole Normale Supérieure de Lyon (France); P. Böhm, Leibniz-Institut für Astrophysik (Germany)

8451 OC Significantly reducing the processing times of high-speed photometry data sets using a distributed computing model [8451-11]

P. Doyle, F. Mtenzi, Dublin Institute of Technology (Ireland); N. Smith, A. Collins, Cork Institute of Technology (Ireland); B. O'Shea, Dublin Institute of Technology (Ireland)

8451 OD The Dark Energy Survey data processing and calibration system [8451-12]

J. J. Mohr, Ludwig-Maximilians-Univ. München (Germany) and Max-Planck-Institut für extraterrestrische Physik (Germany); R. Armstrong, Univ. of Pennsylvania (United States); E. Bertin, Institut d'Astrophysique de Paris (France); G. Daves, National Ctr. for Supercomputing Applications, Univ. of Illinois at Urbana-Champaign (United States); S. Desai, Ludwig-Maximilians-Univ. München (Germany); M. Gower, National Ctr. for Supercomputing Applications, Univ. of Illinois at Urbana-Champaign (United States); R. Gruendl, W. Hanlon, Univ. of Illinois at Urbana-Champaign (United States); N. Kuropatkin, H. Lin, J. Marriner, Fermi National Accelerator Lab. (United States); D. Petravic, National Ctr. for Supercomputing Applications, Univ. of Illinois at Urbana-Champaign (United States); I. Sevilla, Ctr. de Investigaciones Energéticas, Medioambientales y Tecnológicas (Spain); M. Swanson, Smithsonian Astrophysical Observatory, Harvard Smithsonian Ctr. for Astrophysics (United States); T. Tomashek, National Ctr. for Supercomputing Applications, Univ. of Illinois at Urbana-Champaign (United States); D. Tucker, B. Yanny, Fermi National Accelerator Lab. (United States)

8451 OE The Italian DPC: infrastructure and operations for the Italian contribution to the Gaia data processing and analysis consortium [8451-13]

R. Messineo, ALTEC S.p.A. (Italy); R. Morbidelli, INAF - Osservatorio Astrofisico di Torino (Italy); M. Martino, E. Pigozzi, A. F. Mulone, ALTEC S.p.A. (Italy); A. Vecchiato, INAF - Osservatorio Astrofisico di Torino (Italy) 
8451 OF Automated and generalized integral-field spectroscopy data reduction using P3D [8451-14]

C. Sandin, P. Weilbacher, F. Tabataba-Vakili, S. Kamann, O. Streicher, Leibniz-Institut für

Astrophysik (Germany)

\section{CONTROL SYSTEMS I}

8451 OG Service-oriented architecture for the ARGOS instrument control software [8451-88] J. Borelli, Max-Planck-Institut für Astronomie (Germany); L. Barl, Max-Planck-Institut für extraterrestrische Physik (Germany); W. Gässler, M. Kulas, Max-Planck-Institut für Astronomie (Germany); S. Rabien, Max-Planck-Institut für extraterrestrische Physik (Germany)

$8451 \mathrm{OH}$ Development of the ACS+OPC UA based control system for a CTA medium size telescope prototype [8451-16]

B. Behera, Deutsches Elektronen-Synchrotron (Germany); I. Oya, E. Birsin, Humboldt Univ. (Germany); H. Köppel, D. Melkumyan, S. Schlenstedt, Deutsches Elektronen-Synchrotron (Germany); T. Schmidt, High Tech Consulting (Germany); U. Schwanke, Humboldt Univ. (Germany); P. Wegner, S. Wiesand, M. Winde, Deutsches Elektronen-Synchrotron (Germany)

8451 Ol Software control of the Advanced Technology Solar Telescope enclosure PLC hardware using COTS software [8451-17]

A. J. Borrowman, Observatory Sciences Ltd. (United Kingdom); L. de Bilbao, J. Ariño, G. Murga, IDOM (Spain); B. Goodrich, J. R. Hubbard, National Solar Observatory (United States); A. Greer, C. Mayer, P. Taylor, Observatory Sciences Ltd. (United Kingdom)

8451 0J Simultaneous control of multiple instruments at the Advanced Technology Solar Telescope [8451-18]

E. M. Johansson, B. Goodrich, National Solar Observatory (United States)

8451 OK The STELLA robotic observatory on Tenerife [8451-19]

M. Weber, T. Granzer, K. G. Strassmeier, Leibniz-Institut für Astrophysik (Germany)

\section{COMPUTING INNOVATIONS}

$8451 \mathrm{OL}$ Design and implementation of a distributed system for the PAUCam camera control system [8451-104]

O. Ballester, C. Pio, C. Hernández-Ferrer, Institut de Física d'Altes Energies (Spain); N. Tonello, Port d'Informació Científica (Spain); S. Serrano, Institut d'Estudis Espacials de Catalunya, CSIC (Spain)

8451 OM Towards dynamic light-curve catalogues (Invited Paper) [8451-21]

B. Scheers, Astronomical Institute Anton Pannekoek, Univ. of Amsterdam (Netherlands) and Ctr. Wiskunde \& Informatica (Netherlands); F. Groffen, Ctr. Wiskunde \& Informatica (Netherlands)

8451 ON Design and implementation of a general main axis controller for the ESO telescopes [8451-134]

S. Sandrock, N. Di Lieto, L. Pettazzi, T. Erm, European Southern Observatory (Germany) 
845100 A standard framework for developing instrument controllers for the ATST [8451-95] J. R. Hubbard, E. M. Johansson, National Solar Observatory (United States)

8451 OP UAF: a generic OPC unified architecture framework [8451-36]

W. Pessemier, G. Deconinck, G. Raskin, Katholieke Univ. Leuven (Belgium); P. Saey, Katholieke Univ. Leuven (Belgium) and Katholieke Hogeschool St.-Lieven (Belgium); $\mathrm{H}$. Van Winckel, Katholieke Univ. Leuven (Belgium)

CONTROL SYSTEMS II

$84510 Q$ The last mile of the ALMA software development: lessons learned (Invited Paper) [8451-25] A. M. Chavan, European Southern Observatory (Germany); B. E. Glendenning, National Radio Astronomy Observatory (United States); J. Ibsen, Atacama Large Millimeter/submillimeter Array (Chile); J. Kern, National Radio Astronomy Observatory (United States); G. Kosugi, National Astronomical Observatory of Japan (Japan); G. Raffi, Atacama Large Millimeter/submillimeter Array (Chile); E. Schmid, J. Schwarz, European Southern Observatory (Germany)

8451 OR Adoption of new software and hardware solutions at the VLT: the ESPRESSO control architecture case [8451-26]

R. Cirami, P. Di Marcantonio, I. Coretti, P. Santin, M. Mannetta, V. Baldini, S. Cristiani, INAF Osservatorio Astronomico di Trieste (Italy); M. Abreu, A. Cabral, Univ. of Lisbon (Portugal); M. Monteiro, Univ. do Porto (Portugal); D. Mégevand, Observatoire Astronomique de l'Univ. de Genève (Switzerland); F. Zerbi, INAF - Osservatorio Astronomico di Brera (Italy)

8451 OS Conceptual design of the control software for the European Solar Telescope [8451-27] P. Di Marcantonio, R. Cirami, INAF - Osservatorio Astronomico di Trieste (Italy); P. Romano, R. Cosentino, INAF - Osservatorio Astrofisico di Catania (Italy); I. Ermolli, F. Giorgi, INAF Osservatorio Astronomico di Roma (Italy)

8451 OT South Pole Telescope software systems: control, monitoring, and data acquisition [8451-28] K. Story, E. Leitch, The Univ. of Chicago (United States); P. Ade, Cardiff Univ. (United Kingdom); K. A. Aird, Kavli Institute for Cosmological Physics, The Univ. of Chicago (United States); J. E. Austermann, Univ. of Colorado at Boulder (United States); J. A. Beall, D. Becker, National Institute of Standards and Technology (United States); A. N. Bender, McGill Univ. (Canada); B. A. Benson, L. E. Bleem, Kavli Institute for Cosmological Physics, The Univ. of Chicago (United States); J. Britton, National Institute of Standards and Technology (United States); J. E. Carlstrom, C. L. Chang, Kavli Institute for Cosmological Physics, The Univ. of Chicago (United States) and Argonne National Lab. (United States); H. C. Chiang, Kavli Institute for Cosmological Physics, The Univ. of Chicago (United States); H.-M. Cho, National Institute of Standards and Technology (United States); T. M. Crawford, A. T. Crites, Kavli Institute for Cosmological Physics, The Univ. of Chicago (United States); A. Datesman, Argonne National Lab. (United States); T. de Haan, M. A. Dobbs, McGill Univ. (Canada); W. Everett, A. Ewall-Wice, Kavli Institute for Cosmological Physics, The Univ. of Chicago (United States); E. M. George, Univ. of California, Berkeley (United States); N. W. Halverson, Univ. of Colorado at Boulder (United States); N. Harrington, Univ. of California, Berkeley (United States); J. W. Henning, Univ. of Colorado at Boulder (United States); G. C. Hilton, National Institute of Standards and Technology (United States); W. L. Holzapfel, Univ. of California, Berkeley (United States); S. Hoover, N. Huang, Kavli Institute for Cosmological Physics, The Univ. of Chicago (United States); J. Hubmayr, K. D. Irwin, National Institute of Standards and Technology (United States); M. Karfunkle, R. Keisler, Kavli Institute for Cosmological Physics, The Univ. of Chicago (United States); J. Kennedy, McGill Univ. (Canada); A. T. Lee, Univ. of California, Berkeley (United States); 
D. Li, National Institute of Standards and Technology (United States); M. Lueker, California Institute of Technology (United States); D. P. Marrone, Steward Observatory, Univ. of Arizona (United States); J. J. McMahon, Univ. of Michigan (United States); J. Mehl, S. S. Meyer, J. Montgomery, Kavli Institute for Cosmological Physics, The Univ. of Chicago (United States); T. E. Montroy, J. Nagy, Case Western Reserve Univ. (United States); T. Natoli, The Univ. of Chicago (United States); J. P. Nibarger, M. D. Niemack, National Institute of Standards and Technology (United States); V. Novosad, Argonne National Lab. (United States); S. Padin, Kavli Institute for Cosmological Physics, The Univ. of Chicago (United States); C. Pryke, Univ. of Minnesota (United States); C. L. Reichardt, Univ. of California, Berkeley (United States); J. E. Ruhl, B. R. Saliwanchik, J. T. Sayre, Case Western Reserve Univ. (United States); K. K. Schaffer, The School of the Art Institute of Chicago (United States); E. Shirokoff, California Institute of Technology (United States); G. Smecher, McGill Univ. (Canada); B. Stalder, Harvard-Smithsonian Ctr. for Astrophysics (United States); C. Tucker, Cardiff Univ. (United Kingdom); K. Vanderlinde, McGill Univ. (Canada); J. D. Vieira, California Institute of Technology (United States); G. Wang, Argonne National Lab. (United States); R. Williamson, Kavli Institute for Cosmological Physics, The Univ. of Chicago (United States); V. Yefremenko, Argonne National Lab. (United States); K. W. Yoon, National Institute of Standards and Technology (United States); E. Young, Univ. of California, Berkeley (United States)

8451 OU A modern approach to upgrading the telescope control system of the CTIO Blanco 4-m telescope [8451-29]

M. Warner, R. Cantarutti, G. Schumacher, E. Mondaca, O. Estay, M. Martinez, V. Aguirre, R. Alvarez, R. Leiva, T. M. C. Abbott, N. S. van der Bliek, Cerro Tololo Inter-American Observatory (Chile)

\section{CYBERINFRASTRUCTURE AND HPC}

8451 OV Data management cyberinfrastructure for the Large Synoptic Survey Telescope (Invited Paper) [8451-30]

D. M. Freemon, National Ctr. for Supercomputing Applications, Univ. of Illinois at UrbanaChampaign (United States); K.-T. Lim, J. Becla, G. P. Dubois-Felsman, SLAC National Accelerator Lab. (United States); J. Kantor, LSST Corp. (United States)

8451 OW ALMA software scalability experience with growing number of antennas [8451-31] M. Mora, Associated Universities, Inc. (Chile); J. Avarias, National Radio Astronomy Observatory (United States); A. Tejeda, J. P. Gil, Associated Universities, Inc. (Chile); H. Sommer, European Southern Observatory (Germany)

8451 0X The DIRP framework: flexible HPC based post-processing of TB size datasets [8451-32] A. Wicenec, International Ctr. for Radio Astronomy Research, The Univ. of Western Australia (Australia); C. Harris, iVEC, The Univ. of Western Australia (Australia); K. Vinsen, P. Quinn, International Ctr. for Radio Astronomy Research, The Univ. of Western Australia (Australia)

8451 OY A complete history of everything [8451-33]

K. Lanclos, W. T. S. Deich, Univ. of California Observatories, Lick Observatory (United States)

$84510 Z$ UKIRT remote operations fail-safe system [8451-34]

B. Gorges, C. Walther, T. Chuter, Joint Astronomy Ctr. (United States) 
845110 Interaction design challenges and solutions for ALMA operations monitoring and control (Invited Paper) [8451-35]

E. Pietriga, INRIA (France) and INRIA Chile - CIRIC (Chile); P. Cubaud, Conservatoire National des Arts Métiers (France); J. Schwarz, European Southern Observatory (Germany); R. Primet, INRIA (France); M. Schilling, European Southern Observatory (Germany); D. Barkats, E. Barrios, B. Vila Vilaro, Joint ALMA Office (Chile)

845111 GMT software and controls overview [8451-131]

J. M. Filgueira, M. Bec, J. Soto, N. Liu, C. Y. Peng, GMT Corp. (United States)

845112 The readout and control system of the Dark Energy Camera [8451-37]

K. Honscheid, A. Elliott, The Ohio State Univ. (United States); J. Annis, Fermi National Accelerator Lab. (United States); M. Bonati, National Optical Astronomy Observatories (United States); E. Buckley-Geer, Fermi National Accelerator Lab. (United States); F. Castander, Institut d'Estudis Espacials de Catalunya (Spain); L. daCosta, A. Fausti, Observatorio National (Brazil); I. Karliner, Univ. of Illinois (United States); S. Kuhlmann, Argonne National Lab. (United States); E. Neilsen, Fermi National Accelerator Lab. (United States); K. Patton, The Ohio State Univ. (United States); K. Reil, A. Roodman, SLAC National Accelerator Lab. (United States); J. Thaler, Univ. of Illinois (United States); S. Serrano, Institut d'Estudis Espacials de Catalunya (Spain); M. Soares Santos, Fermi National Accelerator Lab. (United States); E. Suchyta, The Ohio State Univ. (United States)

845113 Instrument control software for the Visible Broadband Imager using ATST common services framework and base [8451-38]

A. Ferayorni, National Solar Observatory (United States)

845114 Intercontinental network control platform and robotic observation for Chinese Antarctic telescopes [8451-39]

L. Xu, Nanjing Institute of Astronomical Optics \& Technology (China) and Key Lab. of Astronomical Optics \& Technology, Nanjing Institute of Astronomical Optics \& Technology (China)

\section{DATA MANAGEMENT, PIPELINES, AND ARCHIVES}

845115 A distributed data management system for data-intensive radio astronomy (Invited Paper) [8451-40]

A. Grimstrup, Univ. of Calgary (Canada); V. Mahadevan, Univ. of British Columbia Okanagan (Canada); O. Eymere, IBM Canada Ltd. (Canada); K. Anderson, Univ. of British Columbia Okanagan (Canada); C. Kiddle, R. Simmonds, Univ. of Calgary (Canada); E. Rosolowsky, Univ. of British Columbia Okanagan (Canada); A. R. Taylor, Univ. of Calgary (Canada)

845116 J-PAS data management pipeline and archiving [8451-41]

D. Cristóbal-Hornillos, N. Gruel, J. Varela, A. López-Sainz, A. Ederoclite, Ctr. de Estudios de Física del Cosmos de Aragón (Spain); M. Moles, Ctr. de Estudios de Física del Cosmos de Aragón (Spain) and Instituto de Astrofísica de Andalucía (Spain); A. J. Cenarro, A. MarínFranch, J. Hernández-Fuertes, A. Yanes-Díaz, S. Chueca, S. Rueda-Tervel, F. Rueda-Tervel, R. Luis-Simoes, Ctr. de Estudios de Física del Cosmos de Aragón (Spain) 
845117 The LOFAR long-term archive: e-infrastructure on petabyte scale [8451-42] H. Holties, A. Renting, Y. Grange, ASTRON (Netherlands)

845118 The MWA archive infrastructure: archiving terabytes of data over dedicated WAN connections [8451-43]

A. Wicenec, The Univ. of Western Australia (Australia); D. Pallot, Curtin Univ. (Australia);

A. Checcucci, S. Kitaeff, K. Vinsen, C. Wu, The Univ. of Western Australia (Australia)

845119 ESO Archive data and metadata model [8451-44]

A. Dobrzycki, C. da Rocha, I. Vera, M. H. Vuong, T. Bierwirth, V. Forchì, N. Fourniol, C. Moins,

S. Zampieri, European Southern Observatory (Germany)

\section{OBSERVING AND IMAGING}

8451 1A The ALMA OT in early science: supporting multiple customers (Invited Paper) [8451-45]

A. Bridger, S. Williams, S. McLay, UK Astronomy Technology Ctr. (United Kingdom);

H. Yatagai, National Astronomical Observatory of Japan (Japan); M. Schilling, A. Biggs,

R. Tobar, R. H. Warmels, European Southern Observatory (Germany)

8451 1B Evolution of the phase 2 preparation and observation tools at ESO [8451-46]

D. Dorigo, European Southern Observatory (Germany); B. Amarand, top itservices AG

(Germany); T. Bierwirth, Y. Jung, P. Santos, F. Sogni, I. Vera, European Southern Observatory (Germany)

8451 1C Accelerated speckle imaging with the ATST visible broadband imager [8451-47]

F. Wöger, A. Ferayorni, National Solar Observatory (United States)

8451 1E Advanced PANIC quick-look tool using Python [8451-49]

J.-M. Ibáñez, A. J. García Segura, Instituto de Astrofísica de Andalucía, CSIC (Spain);

C. Storz, J. W. Fried, Max-Planck-Institut für Astronomie (Germany); M. Fernández,

J. F. Rodríguez Gómez, V. Terrón, M. C. Cárdenas, Instituto de Astrofísica de Andalucía,

CSIC (Spain)

\section{COMMUNICATIONS, COLLABORATION, AND COORDINATION}

8451 IF Astro-WISE information system [8451-50]

E. A. Valentijn, A. N. Belikov, G. A. Verdoes Kleijn, Univ. of Groningen (Netherlands);

O. R. Williams, Donald Smits Ctr. For Information Technology, Univ. of Groningen

(Netherlands) 
8451 IG EVALSO, a high-bandwidth communication infrastructure to efficiently connect the ESO Paranal and the Cerro Armazones Observatories to Europe: demonstration activities and start of operations [8451-51]

R. Lemke, Ruhr-Univ. Bochum (Germany); F. Comeron, European Southern Observatory (Germany); R. Chini, Ruhr-Univ. Bochum (Germany); G. Filippi, European Southern Observatory (Germany); J. Emerson, Queen Mary Univ. of London (United Kingdom); K. Kuijken, Leiden Observatory, Leiden Univ. (Netherlands); D. Dobrzycka, European Southern Observatory (Germany); A. Wright, European Southern Observatory (Chile); S. Zampieri, European Southern Observatory (Germany); F. Liello, Univ. degli Studi di Trieste (Italy)

$8451 \mathrm{lH}$ Enabling efficient electronic collaboration between LIGO and other astronomy communities using federated identity and COmanage [8451-52]

H. Flanagan, Internet2 (United States); M. Huynh, Univ. of Wisconsin, Milwaukee (United States); K. Klingenstein, Internet2 (United States); S. Koranda, Univ. of Wisconsin, Milwaukee (United States); B. Oshrin, Internet2 (United States)

845111 REMOTES: reliable and modular telescope solution for seamless operation and monitoring of various observation facilities [8451-53]

M. Jakubec, Astronomical Institute of the Academy of Sciences of the Czech Republic (Czech Republic) and Czech Technical Univ. Prague (Czech Republic); P. Skala,

M. Sedlacek, M. Nekola, J. Strobl, Astronomical Institute of the Academy of Sciences of the Czech Republic (Czech Republic); M. Blazek, R. Hudec, Astronomical Institute of the Academy of Sciences of the Czech Republic (Czech Republic) and Czech Technical Univ. Prague (Czech Republic)

8451 1 J A symbiotic relationship between HST and JWST operations software systems development [8451-54]

D. C. Taylor, M. Bertch, R. E. Douglas, Jr., M. Giuliano, A. Roman, Space Telescope Science Institute (United States)

\section{Part 2}

\section{POSTER SESSION}

$8451 \mathrm{lL}$ The EMIR experience in the use of software control simulators to speed up the time to telescope [8451-2]

P. Lopez Ramos, Instituto de Astrofísica de Canarias (Spain); J. C. López-Ruiz, Instituto de Astrofísica de Canarias (Spain) and Univ. de la Laguna (Spain); H. Moreno Arce, J. Rosich, J. M. Perez Menor, Instituto de Astrofísica de Canarias (Spain)

$84511 \mathrm{M}$ More flexibility in representing geometric distortion in astronomical images [8451-57] D. L. Shupe, NASA Herschel Science Ctr., California Institute of Technology (United States): R. R. Laher, L. Storrie-Lombardi, J. Surace, C. Grillmair, Spitzer Science Ctr., California Institute of Technology (United States); D. Levitan, B. Sesar, California Institute of Technology (United States) 
8451 iN Virtualization in network and servers infrastructure to support dynamic system reconfiguration in ALMA [8451-58]

T.-C. Shen, N. Ovando, M. Bartsch, M. Simmond, G. Vélez, M. Robles, R. Soto, Atacama Large Millimeter/submillimeter Array (Chile); J. Ibsen, European Southern Observatory (Chile); C. Saldias, Atacama Large Millimeter/submillimeter Array (Chile)

8451 IP Reflector adjustment for a large radio telescope based on active optics [8451-60] T. Li, Nanjing Institute of Astronomical Optics \& Technology (China) and Graduate Univ. of Chinese Academy of Sciences (China); Z. Zhang, A. Li, Y. Wang, Nanjing Institute of Astronomical Optics \& Technology (China)

$84511 Q$ A mask quality control tool for the OSIRIS multi-object spectrograph [8451-61]

J. C. López-Ruiz, Instituto de Astrofísica de Canarias (Spain) and Univ. de la Laguna (Spain); J. J. Vaz Cedillo, Instituto de Astrofísica de Canarias (Spain); A. Ederoclite, Ctr. de Estudios de Física del Cosmos de Aragón (Spain); Á. Bongiovanni, Instituto de Astrofísica de Canarias (Spain) and Univ. de la Laguna (Spain); V. González Escalera, Instituto de Astrofísica de Canarias (Spain)

8451 IR ALMA software regression tests: the evolution under an operational environment [8451-62] R. Soto, V. González, Associated Universities Inc. (Chile); J. Ibsen, European Southern Observatory (Chile); M. Mora, N. Sáez, T.-C. Shen, Associated Universities Inc. (Chile)

8451 is ALMA operation support software and infrastructure [8451-63] T.-C. Shen, R. Soto, M. Mora, J. Reveco, Atacama Large Millimeter/submillimeter Array (Chile); J. Ibsen, European Southern Observatory (Chile)

8451 iT Development of telescope control system for the $50 \mathrm{~cm}$ telescope of UC Observatory Santa Martina [8451-64]

T.-C. Shen, Pontificia Univ. Catolica de Chile (Chile); R. Soto, Univ. Técnica Federico Santa Maria (Chile); J. Reveco, Univ. de Concepción (Chile); L. Vanzi, J. M. Fernández, Pontificia Univ. Catolica de Chile (Chile); P. Escarate, Univ. Técnica Federico Santa Maria (Chile); V. Suc, Pontificia Univ. Catolica de Chile (Chile)

$84511 \mathrm{U}$ The MUSE observation preparation tool [8451-65]

L. Piqueras, J. Richard, R. Bacon, A. Pecontal, Observatoire de Lyon, CNRS, Univ. de Lyon (France), Ctr. de Recherche Astronomique de Lyon (France), and Ecole Normale Supérieure de Lyon (France); P. Baksai, ESO Paranal Observatory (Chile); J. Vernet, European Southern Observatory (Germany)

8451 IV Korea microlensing telescope network: data management plan [8451-66] C.-U. Lee, D.-J. Kim, S.-L. Kim, B.-G. Park, S.-M. Cha, Korea Astronomy and Space Science Institute (Korea, Republic of)

8451 1X HARPS-N: software path from the observation block to the image [8451-68] D. Sosnowska, Observatoire Astronomique de l'Univ. de Genève (Switzerland); M. Lodi, INAF - Telescopio Nazionale Galileo (Spain); X. Gao, UK Astronomy Technology Ctr. (United Kingdom); N. Buchschacher, École Polytechnique Fédérale de Lausanne (Switzerland); A. Vick, UK Astronomy Technology Ctr. (United Kingdom); J. Guerra, M. Gonzalez, INAF Telescopio Nazionale Galileo (Spain); D. Kelly, UK Astronomy Technology Ctr. (United Kingdom); C. Lovis, F. Pepe, Observatoire Astronomique de l'Univ. de Genève (Switzerland); E. Molinari, INAF - Telescopio Nazionale Galileo (Spain); A. C. Cameron, Univ. 
of St. Andrews (United Kingdom); D. Latham, Harvard-Smithsonian Ctr. for Astrophysics (United States); S. Udry, Observatoire Astronomique de l'Univ. de Genève (Switzerland)

8451 IY Research of simulation framework for telescope wireless networks control system [8451-71] X. Shuai, Nanjing Univ. of Science and Technology (China) and Chizhou College (China); H. Qian, Chizhou College (China)

845112 Toolkit of automated database creation and cross-match [8451-72]

Y. Zhang, National Astronomical Observatories (China); H. Zheng, North China Electric Power Univ. (China); T. Pei, Y. Zhao, National Astronomical Observatories (China)

845121 SPHERE instrumentation software: a progress report [8451-74]

A. Baruffolo, D. Fantinel, INAF - Osservatorio Astronomico di Padova (Italy); L. Gluck, Institut de Planétologie et d'Astrophysique de Grenoble (France); B. Salasnich, INAF - Osservatorio Astronomico di Padova (Italy); G. Zins, Institut de Planétologie et d'Astrophysique de Grenoble (France); P. Steiner, ETH Zurich (Switzerland); M. Micallef, Institut de Planétologie et d'Astrophysique de Grenoble (France); P. Bruno, INAF - Osservatorio Astrofisico di Catania (Italy); D. Popovic, R. H. Donaldson, E. Fedrigo, M. Kiekebusch, C. Soenke, M. Suarez Valles, European Southern Observatory (Germany)

845122 Remote monitoring and fault recovery for FPGA-based field controllers of telescope and instruments [8451-75]

Y. Zhu, D. Zhu, Nanjing Univ. of Posts and Telecommunications (China); J. Wang, National Astronomical Observatories, Nanjing Institute of Astronomical Optics \& Technology (China)

845123 Commissioning the VST Telescope control software [8451-76]

P. Schipani, INAF - Osservatorio Astronomico di Capodimonte (Italy); J. Argomedo, European Southern Observatory (Germany); L. Marty, INAF - Osservatorio Astronomico di Capodimonte (Italy)

845124 Using ODGWs with GSAOI: software and firmware implementation challenges [8451-77] P. J. Young, P. McGregor, J. van Harmelen, The Australian National Univ. (Australia); B. Neichel, Gemini Observatory (Chile)

845125 Cure-WISE: HETDEX data reduction with Astro-WISE [8451-78]

J. M. Snigula, Max-Planck-Institut für extraterrestrische Physik (Germany) and Univ.-

Sternwarte München (Germany); M. E. Cornell, The Univ. of Texas at Austin (United States);

N. Drory, Univ. Instituto de Astronmía, Nacional Autónoma de México (Mexico);

M. Fabricius, M. Landriau, Max-Planck-Institut für extraterrestrische Physik (Germany) and Univ.-Sternwarte München (Germany); G. J. Hill, K. Gebhardt, The Univ. of Texas at Austin (United States)

845126 Multiple guide star acquisition software for LINC-NIRVANA [8451-79]

T. Bertram, F. Kittmann, L. Mohr, Max-Planck-Institut für Astronomie (Germany)

845127 OpenROCS: a software tool to control robotic observatories [8451-80]

J. Colomé, J. Sanz, F. Vilardell, I. Ribas, P. Gil, ICE - Institut de Ciències de I'Espai, CSIC (Spain)

845128 The 3,6 m optical telescope for ARIES: the control system [8451-82]

E. Gabriel, C. Bastin, M. Piérard, AMOS s.a. (Belgium) 
845129 Technical solutions in preparing data for the Keck Observatory Archive (KOA) [8451-83] H. D. Tran, J. A. Mader, R. W. Goodrich, M. Tsubota, W. M. Keck Observatory (United States)

$84512 B \quad$ Open source pipeline for ESPaDOnS reduction and analysis [8451-86]

E. Martioli, D. Teeple, N. Manset, D. Devost, K. Withington, Canada-France-Hawaii Telescope (United States); A. Venne, Univ. de Sherbrooke (Canada); M. Tannock, Univ. of Victoria (Canada)

$84512 C \quad$ MESA: Mercator scheduler and archive system [8451-87]

F. Merges, S. Prins, W. Pessemier, G. Raskin, J. Perez Padilla, H. Van Winckel, C. Aerts, Institute for Astronomy, Katholieke Univ. Leuven (Belgium)

$84512 D \quad$ SDAl: a key piece of software to manage the new wideband backend at Robledo [8451-89]

J. R. Rizzo, M. Gutiérrez Bustos, Ctr. de Astrobiología (Spain); T. B. H. Kuiper, Jet Propulsion Lab. (United States); J. Cernicharo, Ctr. de Astrobiología (Spain); I. Sotvela, Madrid Deep Space Communication Complex (Spain); A. Pedreira, Instituto Nacional de Técnica Aeroespacial (Spain)

$84512 \mathrm{E}$ Confronting the numerical simulations of the VLT/MUSE instrument with the first real data [8451-91]

A. Jarno, R. Bacon, A. Pécontal-Rousset, Ctr. de Recherche Astrophysique de Lyon, CNRS, Univ. de Lyon (France); O. Streicher, P. Weilbacher, Leibniz-Institut für Astrophysik (Germany)

$84512 \mathrm{~F}$ The future of the TNG telescope control software [8451-92] J. Guerra, J. San Juan, M. Lodi, N. Hernandez, Fundación Galileo Galilei - INAF (Spain)

$84512 \mathrm{H}$ Software-centric view on the LINC-NIRVANA beam control concept [8451-96] J. Trowitzsch, T. Bertram, Max-Planck-Institut für Astronomie (Germany)

845121 Design of LAMOST data processing and production database [8451-97] Y. Guo, A. Luo, F. Wang, Z. Bai, J. Li, National Astronomical Observatories (China)

$84512 \mathrm{~K}$ The improvement of CCD auto-guiding system for $2.5 \mathrm{~m}$ telescope [8451-99] L. Chen, Nanjing Institute of Astronomical Optics \& Technology (China) and Graduate Univ. of Chinese Academy of Sciences (China); Z. Zhang, H. Wang, Nanjing Institute of Astronomical Optics \& Technology (China)

$84512 \mathrm{~L}$ Diving into the Sardinia Radio Telescope minor servo system [8451-100] M. Buttu, INAF - Osservatorio Astronomico di Cagliari (Italy); A. Orlati, G. Zacchiroli, M. Morsiani, F. Fiocchi, INAF - Istituto di Radioastronomia (Italy); F. Buffa, INAF - Osservatorio Astronomico di Cagliari (Italy); G. Maccaferri, INAF - Istituto di Radioastronomia (Italy); G. P. Vargiu, C. Migoni, S. Poppi, INAF - Osservatorio Astronomico di Cagliari (Italy); S. Righini, INAF - Istituto di Radioastronomia (Italy); A. Melis, INAF - Osservatorio Astronomico di Cagliari (Italy)

$84512 M \quad$ The control software for the Sardinia Radio Telescope [8451-101] A. Orlati, INAF - Istituto di Radioastronomia (Italy); M. Buttu, A. Melis, C. Migoni, S. Poppi, INAF - Osservatorio Astronomico di Cagliari (Italy); S. Righini, INAF - Istituto di Radioastronomia (Italy) 
$84512 \mathrm{~N}$ Open-source framework for documentation of scientific software written on MATLABcompatible programming languages [8451-102]

M. V. Konnik, J. Welsh, The Univ. of Newcastle (Australia)

845120 A motion control networked solution for the PAUCam slow control [8451-103] O. Ballester, C. Pio, C. Hernández-Ferrer, M. Albareda-Sirvent, Institute for High Energy Physics (Spain)

$84512 Q \quad$ GCS component development cycle [8451-106] J. A. Rodríguez, R. Macias, J. Molgo, D. Guerra, M. Pi, GTC Project, Instituto de Astrofísica de Canarias (Spain)

$84512 S \quad$ CARMENES. IV: instrument control software [8451-108]

J. Guàrdia, J. Colomé, I. Ribas, Institut d'Estudis Espacials de Catalunya (Spain); H.-J. Hagen, Hamburger Sternwarte (Germany); R. Morales, M. Abril, Instituto de Astrofísica de Andalucía (Spain); D. Galadí-Enríquez, Ctr. Astronómico Hispano-Alemán (Spain); W. Seifert, Landessternwarte (Germany); M. A. Sánchez Carrasco, Instituto de Astrofísica de Andalucía (Spain); A. Quirrenbach, Landessternwarte (Germany); P. J. Amado, Ctr. de Astrobiología, Instituto de Astrofísica de Andalucía (Spain); J. A. Caballero, Instituto Nacional de Técnica Aeroespacial (Spain); H. Mandel, Landessternwarte (Germany)

$84512 T \quad$ Use of RTS2 for LSST multiple channel CCD characterisation [8451-109] P. Kubánek, M. Prouza, Institute of Physics of the ASCR, v.v.i. (Czech Republic); I. Kotov, P. O'Connor, Brookhaven National Lab. (United States); P. Doherty, Harvard Univ. (United States); J. Frank, Brookhaven National Lab. (United States)

$84512 \mathrm{U} \quad$ A complete solar eruption activities processing tool with robotization and real time (I) [8451-110]

G. Lin, National Astronomical Observatories (China)

$84512 \mathrm{~V}$ Design and first commissioning results of PLC-based control systems for the Mercator Telescope [8451-112]

W. Pessemier, G. Deconinck, G. Raskin, Katholieke Univ. Leuven (Belgium); P. Saey, Katholieke Univ. Leuven (Belgium) and Katholieke Hogeschool St.-Lieven (Belgium); $\mathrm{H}$. Van Winckel, Katholieke Univ. Leuven (Belgium)

$84512 X \quad$ ESPRESSO front-end guiding algorithm [8451-115]

M. Landoni, Univ. degli Studi dell'Insubria (Italy) and INAF - Osservatorio Astronomico di Brera (Italy); M. Riva, F. M. Zerbi, INAF - Osservatorio Astronomico di Brera (Italy); D. Mégevand, Observatoire Astronomique de l'Univ. de Genève (Switzerland); A. Cabral, Univ. of Lisbon (Portugal); S. Cristiani, INAF - Osservatorio Astronomico di Trieste (Italy)

$84512 Y \quad$ Image acquisition system with three CCD cameras [8451-116]

B. Li, Y. Zhang, Kunming Univ. of Science and Technology (China); L. Yang, W. Mao, J. Wang, Yunnan Astronomical Observatories (China)

$84512 Z$ Final redshift determination of LAMOST pilot survey [8451-117]

F. Wang, A.-L. Luo, H. Zhang, Nanjing Institute of Astronomical Optics \& Technology (China) 
845130 Design and realization of the backup field controllers for LAMOST spectrographs [8451-118] J. Wang, Nanjing Institute of Astronomical Optics \& Technology (China); Z. Han, Nanjing Institute of Astronomical Optics \& Technology (China) and Graduate Univ. of Chinese Academy of Sciences (China); Y. Zeng, S. Dai, Z. Hu, Y. Zhu, L. Wang, Y. Hou, Nanjing Institute of Astronomical Optics \& Technology (China)

845132 Design and practice multi-channel real time system on deformation control of optical plate [8451-120]

Y. Zheng, Nanjing Institute of Astronomical Optics \& Technology (China); Y. Wang, Y. Li, Nanjing Institute of Astronomical Optics \& Technology (China) and Graduate Univ. of Chinese Academy of Sciences (China); X. Li, Nanjing Institute of Astronomical Optics \& Technology (China)

845133 Support vector machines for photometric redshift measurement of quasars [8451-121] H. Zheng, North China Electric Power Univ. (China); Y. Zhang, Nanjing Institute of Astronomical Optics \& Technology (China)

845134 Review of techniques for photometric redshift estimation [8451-122]

H. Zheng, North China Electric Power Univ. (China); Y. Zhang, Nanjing Institute of Astronomical Optics \& Technology (China)

845135 Survey of approaches for targeting quasars [8451-123]

Y. Zhang, Y. Zhao, Nanjing Institute of Astronomical Optics \& Technology (China)

845136 Upper computer design for active optics [8451-124]

C. Li, Nanjing Institute of Astronomical Optics \& Technology (China) and Graduate Univ. of Chinese Academy of Sciences (China); G. Wang, Nanjing Institute of Astronomical Optics \& Technology (China); L. Gao, Nanjing Institute of Astronomical Optics \& Technology (China) and Graduate Univ. of Chinese Academy of Sciences (China)

845137 Comparison of different interpolation algorithm in feature-based template matching for stellar parameters analysis [8451-125]

B. Du, A. Luo, J. Zhang, Y. Wu, F. Wang, Nanjing Institute of Astronomical Optics \& Technology, (China)

845139 The optical synthetic aperture image restoration based on the improved maximumlikelihood algorithm [8451-127]

Z. Geng, Q. XU, B. Zhang, Z. Gong, Zhengzhou Institute of Surveying and Mapping (China)

8451 3A Estimate LAMOST hot star's parameters by POLLUX [8451-129]

F. Zuo, A. Luo, J. Zhang, Nanjing Institute of Astronomical Optics \& Technology (China)

8451 3B Data access and analysis system for Gaia data handling during operations at Italian DPC: scientific validation and results monitoring approach in support of the AVU operations [8451-130]

R. Morbidelli, INAF - Osservatorio Astrofisico di Torino (Italy); R. Messineo, ALTEC S.p.A. (Italy);

D. Busonero, A. Riva, A. Vecchiato, INAF - Osservatorio Astrofisico di Torino (Italy) 
$84513 \mathrm{C}$ The global sphere reconstruction for the Gaia mission in the Astrometric Verification Unit [8451-132]

A. Vecchiato, U. Abbas, INAF - Osservatorio Astrofisico di Torino (Italy); M. Bandieramonte, Univ. degli Studi di Catania (Italy) and INAF - Osservatorio Astrofisico di Catania (Italy); U. Becciani, L. Bianchi, INAF - Osservatorio Astrofisico di Torino (Italy) and EURIX (Italy); B. Bucciarelli, D. Busonero, M. G. Lattanzi, INAF - Osservatorio Astrofisico di Torino (Italy); R. Messineo, ALTEC S.p.A. (Italy)

8451 3D Qsys NOC-based MPSOC design for LAMOST Spectrographs [8451-133]

Z. Han, Nanjing Institute of Astronomical Optics \& Technology (China) and Graduate Univ. of Chinese Academy of Sciences (China); J. Wang, Y. Zeng, Nanjing Institute of Astronomical Optics \& Technology, (China)

8451 3F The system software development for prime focus spectrograph on Subaru Telescope [8451-136]

A. Shimono, N. Tamura, H. Sugai, H. Karoji, Kavli Institute for the Physics and Mathematics of the Universe, The Univ. of Tokyo (Japan)

$84513 G$ Test results for the Gemini Planet Imager data reduction pipeline [8451-137]

J. Maire, Dunlap Institute for Astronomy \& Astrophysics, Univ. of Toronto (Canada);

M. D. Perrin, Space Telescope Science Institute (United States); R. Doyon, Univ. de Montréal (Canada); J. Chilcote, J. E. Larkin, J. L. Weiss, Univ. of California, Los Angeles (United States); C. Marois, Herzberg Institute of Astrophysics (Canada); Q. M. Konopacky, M. MillarBlanchaer, J. R. Graham, Dunlap Institute for Astronomy \& Astrophysics, Univ. of Toronto (Canada); J. Dunn, Herzberg Institute of Astrophysics (Canada); R. Galicher, Herzberg Institute of Astrophysics (Canada) and Univ. de Montréal (Canada); F. Marchis, S. J. Wiktorowicz, Univ. of California, Berkeley (United States); K. Labrie, S. J. Thomas, S. J. Goodsell, Gemini Observatory (United States); F. T. Rantakyro, Gemini Observatory (Chile); D. W. Palmer, B. A. Macintosh, Lawrence Livermore National Lab. (United States)

$84513 \mathrm{H} \quad$ Electronics and mechanisms control system for FRIDA (inFRared Imager and Dissector for Adaptive optics) [8451-138]

R. Flores-Meza, S. Cuevas, Univ. Nacional Autónoma de México (Mexico); J. J. Díaz, Instituto de Astrofísica de Canarias (Spain); C. Espejo, C. Keiman, G. Lara, B. Sánchez, Univ. Nacional Autónoma de México (Mexico); J. Uribe, Ctr. de Ingeniería y Desarrollo Industrial (Mexico)

$845131 \quad$ SPIRou @ CFHT: data reduction software and simulation tools [8451-141]

É. Artigau, Univ. de Montréal (Canada); F. Bouchy, Institut d'Astrophysique de Paris, CNRS, Univ. Pierre et Marie Curie (France); X. Delfosse, X. Bonfils, INSU, CNRS, Univ. Joseph Fourier Grenoble (France) and Institut de Planétologie et d'Astrophysique de Grenoble (France); J.-F. Donati, Institut de Recherche en Astrophysique et Planétologie, CNRS, Univ. de Toulouse (France); P. Figueira, Ctr. de Astrofísica, Univ. do Porto (Portugal); K. Thanjavur, Canada-France-Hawaii Telescope (United States); D. Lafrenière, R. Doyon, Univ. de Montréal (Canada); C. Surace, C. Moutou, CeSAM, Lab. Astrophysique de Marseille (France) and Observatoire Astronomique de Marseille-Provence, CNRS (France); I. Boisse, Ctr. de Astrofísica, Univ. do Porto (Portugal); L. Saddlemyer, Herzberg Institute of Astrophysics (Canada) and National Research Council of Canada (Canada); D. Loop, Herzberg Institute of Astrophysics (Canada); D. Kouach, Institut de Recherche en Astrophysique et Planétologie, CNRS (France) and Univ. de Toulouse (France); F. Pepe, C. Lovis, Observatoire Astronomique de l'Univ. de Genève (Switzerland); O. Hernandez, 
Univ. de Montréal (Canada); S.-Y. Wang, Institute of Astronomy and Astrophysics, National Taiwan Univ. (Taiwan)

8451 3J SPIRou @ CFHT: design of the instrument control system [8451-142]

G. A. Barrick, T. Vermeulen, Canada-France-Hawaii Telescope (United States);

S. Baratchart, Institut de Recherche en Astrophysique et Planétologie (France);

V. A. Reshetov, Herzberg Institute of Astrophisics (Canada); S.-Y. Wang, Institute of Astronomy and Astrophysics, National Taiwan Univ. (Taiwan); F. Dolon, Observatoire de Haute Provence, Institut Pytheas (France); O. Hernandez, Univ. de Montréal (Canada); F. Pepe, Observatoire Astronomique de I'Univ. de Genève (Switzerland); F. Bouchy, Lab. d'Astrophysique de Marseille (France); J. Dunn, Herzberg Institute of Astrophysics (Canada); M. Dupieux, G. Gallou, M. Larrieu, A. Fonteneau, Institut de Recherche en Astrophysique et Planétologie (France); F. Moreau, Observatoire de Haute Provence, Institut Pytheas (France); F. Wildi, Observatoire de Genève (Switzerland); L. P. Parès, Institut de Recherche en Astrophysique et Planétologie (France); J. N. Thomas, Canada-FranceHawaii Telescope (United States); C.-H. Yan, Institute of Astronomy and Astrophysics, National Taiwan Univ. (Taiwan); R. Doyon, Univ. de Montréal (Canada); J.-F. Donati, Institut de Recherche en Astrophysique et Planétologie (France); P. Vallée, É. Artigau, Univ. de Montréal (Canada); X. Delfosse, P. Rabou, Institut de Planétologie et d'Astrophysique de Grenoble (France); S. Thibault, Univ. Laval (Canada); D. Kouach, Institut de Recherche en Astrophysique et Planétologie (France); D. Loop, Herzberg Institute of Astrophysics (Canada)

8451 3K ORBS: a data reduction software for the imaging Fourier transform spectrometers SpIOMM and SITELLE [8451-143]

T. Martin, L. Drissen, G. Joncas, Univ. Laval (Canada)

Author Index 
Proc. of SPIE Vol. $8451845101-18$

Downloaded From: https://www.spiedigitallibrary.org/conference-proceedings-of-spie on 26 Apr 2023 Terms of Use: https://www.spiedigitallibrary.org/terms-of-use 


\title{
Conference Committee
}

\author{
Symposium Chairs \\ Mark M. Casali, European the Southern Observatory (Germany) \\ Kathryn A. Flanagan, Space Telescope Science Institute (United \\ States) \\ Symposium Cochairs
}

Gillian S. Wright, UK Astronomy Technology Centre (United Kingdom)

Luc Smiard, National Research Council Canada (Canada

Conference Chairs

Nicole M. Radziwill, James Madison University (United States)

Gianluca Chiozzi, European Southern Observatory (Germany)

Conference Program Committee

Alan Bridger, UK Astronomy Technology Centre (United Kingdom)

Kim K. Gillies, Thirty Meter Telescope Observatory Corporation (United States)

Bret Goodrich, National Solar Observatory, ATST (United States)

Juan C. Guzman, ASKAP, Commonwealth Scientific and Industrial Research Organisation (Australia)

Hilton A. Lewis, W. M. Keck Observatory (United States)

David L. Terrett, Rutherford Appleton Laboratory (United Kingdom)

Session Chairs

1 Innovations

Gianluca Chiozzi, European Southern Observatory (Germany)

2 Software Engineering

David L. Terrett, Rutherford Appleton Laboratory (United Kingdom)

3 Data Management and Processing

Erik M. Johansson, National Solar Observatory (United States)

4 Control Systems I

David L. Terrett, Rutherford Appleton Laboratory (United Kingdom) 
5 Computing Innovations

Juan C. Guzman, ASKAP, Commonwealth Scientific and Industrial Research Organisation (Australia)

6 Control Systems II

Hilton A. Lewis, W. M. Keck Observatory (United States)

$7 \quad$ Lightning Talks

Kim K. Gillies, Thirty Meter Telescope Observatory Corporation (United States)

8 Cyberinfrastructure and HPC

Erik M. Johansson, National Solar Observatory (United States)

9 Control Systems III

Juan C. Guzman, ASKAP, Commonwealth Scientific and Industrial Research Organisation (Australia)

10 Data Management, Pipelines, and Archives

Alan Bridger, UK Astronomy Technology Centre (United Kingdom)

11 Observing and Imaging

Gianluca Chiozzi, European Southern Observatory (Germany)

12 Communications, Collaboration, and Coordination

Hilton A. Lewis, W. M. Keck Observatory (United States)

13 New Directions

Alan Bridger, UK Astronomy Technology Centre (United Kingdom) 